\title{
QUALIDADE DAS PRESCRIÇÕES DE MEDICAMENTOS DE CONTROLE ESPECIAL EM UM MUNICÍPIO GAÚCHO
}

QUALITY OF THE REQUIREMENTS OF CONTROLLED DRUGS IN A MUNICIPALITY GAUCHO

LA CALIDAD DE LOS REQUISITOS DE DROGAS CONTROLADAS EN UN MUNICIPIO GAUCHO

\section{Luciano Henrique Pinto ${ }^{1 *}$, Luana Soares Schulter ${ }^{1}$, Rafaela Sierth $^{1}$, Heloisa Biff ${ }^{1}$, Lineu Del Ciampo', Gilmar Sidnei Erzinger $^{1}$}

\author{
${ }^{1}$ Universidade da Região de Joinville - UNIVILLE. Rua Paulo Malschitzki, 10 - \\ Bom Retiro, Joinville - SC - CEP: 89219-710. Telefone/ Fax: +55 47 3461- \\ 9000. \\ E-mail: lucianohp.pq@gmail.com
}

Submetido em 29/08/2014; Aceito em: 03/04/2015

\section{RESUMO}

O objetivo foi verificar a qualidade da prática de prescrição e o cumprimento da legislação conforme a Portaria 344/98 em um município gaúcho, comparando o desempenho das prescrições entre serviço público e privado. Foi realizado um estudo descritivo, transversal, quantitativo e retrospectivo. Os dados foram coletados em prescrições e notificações de duas farmácias de naturezas jurídicas distintas. As variáveis incluem aspectos clínico-demográficos, legibilidade, cumprimento dos procedimentos legais e erros de prescrição, conforme as boas práticas de prescrição estabelecidas pela Organização Mundial da Saúde. Conforme 


\section{- Revista Eletrônica}

\section{RODRIGUES et al.}

REF-ISSN1808-0804 Vol.XII (1), 44-59, 2015.

o fluxo mensal de pacientes foram utilizadas 330 prescrições (120-farmácia pública e 210-farmácia privada). Para análise dos resultados foi realizada estatística descritiva e inferencial. Considerando os três grupos de variáveis (legibilidade, cumprimento dos procedimentos legais e aspectos técnicos) houve 39,4\% de prescrições adequadas. Este desempenho foi melhor na farmácia privada, na qual os erros mais frequentes foram relativos ao cumprimento dos procedimentos legais. A legibilidade das prescrições teve um desempenho superior a de outros estudos, embora parte dos receituários não puderam ser decifrados, podendo comprometer o entendimento do paciente ou do profissional responsável pela dispensação. A qualidade das prescrições de medicamentos controlados dispensados no município avaliado está comprometida, especialmente pelo descumprimento de exigências legais.

Palavras-chave: Psicotrópicos; uso racional de medicamentos; prescrições de medicamentos; erros nas prescrições.

\section{ABSTRACT}

The aim was to assess the quality of prescribing practices and compliance with legislation as Portaria 344/98 in a gaucho municipality, comparing the performance of prescriptions between public and private service. A descriptive, cross-sectional, quantitative and retrospective study. Data were collected on prescriptions and reports of two pharmacies in different legal natures. Variables include clinical and demographic aspects, readability, statutory procedures and prescription errors, according to good prescribing practices established by the World Health Organization. According to the monthly flow of 330 patients prescriptions (120public were used and 210- private pharmacy). For data analysis descriptive and inferential statistics were performed. Considering the three groups of variables (readability, compliance with legal procedures and technical aspects) was $39.4 \%$ of appropriate prescriptions. This performance was better in the private pharmacy, in which the most frequent errors were related to statutory procedures. The legibility of prescriptions had a superior performance to that of other studies, although part of the prescription could not be deciphered, compromising the understanding of the patient or the professional responsible for dispensing. The quality of prescriptions for controlled medications dispensed in the municipality is assessed compromised, especially by non-compliance with legal requirements. 
RODRIGUES et al.

REF-ISSN1808-0804 Vol.XII (1), 44-59, 2015.

Key-words: Psychotropics; rational use of medicines; drug prescriptions; errors in prescriptions.

\section{RESUMEN}

El objetivo fue evaluar la calidad de las prácticas de prescripción y el cumplimiento de la legislación como la Portaria $344 / 98$ en un municipio gaucho, comparar el rendimiento entre el servicio público y privado. Estudio descriptivo, transversal y cuantitativo. Los datos fueron recolectados en las recetas y los informes de farmacias de diferentes naturalezas jurídicas. Las variables incluyen aspectos clínicos/demográficos, la legibilidad, los procedimientos legales y los errores de prescripción, de acuerdo con las buenas prácticas establecidos por la OMS. Para el análisis de los datos de 330 pacientes las recetas se realizaron estadística descriptiva e inferencial. Teniendo en cuenta los tres grupos de variables (legibilidad, cumplimiento de los procedimientos legales y aspectos técnicos) fue $39,4 \%$ de las prescripciones correspondientes. Este desempeño fue mejor en la farmacia privada, en la que los errores más frecuentes se relacionaron con los procedimientos reglamentarios. La legibilidad de las recetas tenía un rendimiento superior a la de otros estudios, aunque parte de la receta no podría ser descifrado, comprometiendo la comprensión de la paciente o el profesional responsable de la dispensación. La calidad de las recetas de medicamentos controlados dispensados en el municipio se evalúa en peligro, especialmente por el incumplimiento de requisitos legales.

Palabras clave: psicotrópicos; uso racional de los medicamentos; recetas de medicamentos; errores en las recetas.

\section{INTRODUÇÃO}

A legislação que aprova o regulamento técnico sobre substâncias e medicamentos sujeitos a controle especial no Brasil é a Portaria n. 0 344/98, da Secretaria de
Vigilância em Saúde/ Ministério da Saúde, de 12 de maio de 1998, que define as seguintes listas de substâncias: A1 e A2 (entorpecentes), A3, B1 e B2 (psicotrópicas), C1 (outras substâncias sujeitas a controle especial), C2 (retinóicas para uso 


\section{- Revista Eletrônica}

\section{RODRIGUES et al.}

REF-ISSN1808-0804 Vol.XII (1), 44-59, 2015.

sistêmico) e C3 (imunossupressoras). Esta portaria normatiza vários parâmetros para a prescrição e venda destes produtos ${ }^{(1)}$.

A Notificação de Receita é o documento que, acompanhado da prescrição, autoriza a dispensação de medicamentos a base de substâncias constantes nesta Portaria. Ambas deverão estar preenchidas de forma legível e a farmácia ou drogaria somente poderá dispensar o medicamento quando todos os itens estiverem devidamente preenchidos. Para as substâncias da lista C1 outras exigências estão detalhadas na legislação como, por exemplo, validade da receita de 30 (trinta) dias contados a partir da data da emissão(1).

Sabe-se que a prescrição é a comunicação entre médico, farmacêutico, enfermeiro, cuidador e paciente $^{(2)}$. Para ser adequada e de qualidade, além de clara e legível, deve seguir os critérios da Organização Mundial da Saúde, que são nome, endereço, telefone do profissional prescritor; data da prescrição; nome e dose do medicamento; forma farmacêutica e quantidade total; iniciais ou assinatura do profissional prescritor; nome, endereço e idade do paciente; rótulo com instruções e cuidados.

Além disso, conforme disposto na Lei $13.909 / 2012$, o profissional da saúde fica obrigado a expedir a prescrição digitada, datilografada ou escrita manual em letra de imprensa, de forma ou em caixa alta. Impõe, ainda, que o profissional da saúde deve orientar o paciente com relação ao uso dos medicamentos prescritos e possíveis efeitos colaterais e determina que os códigos e abreviaturas somente possam ser usados se estiverem contemplados no Código Internacional de Doenças CID.

Logo, quando não cumpridos estes preceitos, podem ocorrer erros nas prescrições ocasionando aos pacientes situações de desconforto devido a dispensação incorreta e/ou em dose inadequada. Além disso, podem ser dispensados medicamentos cujas receitas estão com data adulterada, se este campo não for preenchido pelo médico responsável.

Especialmente quando se trata de psicofármacos, os erros de prescrição são graves, já que estes medicamentos podem causar dependência física e/ou psíquica. A dependência psíquica favorece $\mathrm{O}$ desenvolvimento da procura 


\section{•) Revista Eletrônica}

\section{RODRIGUES et al.}

REF-ISSN1808-0804 Vol.XII (1), 44-59, 2015.

compulsiva pelo medicamento, e a dependência física, é a necessidade continua de tomar o medicamento. De qualquer uma das dependências pode surgir o vício, levando o paciente e os que convivem com ele a ter dificuldade de relacionamento, pois o vicio pode prejudicar o seu comportamento social ${ }^{(3-4)}$.

Segundo a OMS os psicotrópicos podem ser classificados em: ansiolíticos, que diminuem a ansiedade; sedativos, que produzem efeito calmante ou relaxante; antipsicóticos, também chamados de neurolépticos, que são utilizados para aliviar os sintomas da esquizofrenia; antidepressivos, que diminuem os sintomas da depressão; estimulantes psicomotores, conhecidos como psicoestimulantes que causam vigília e euforia ${ }^{(3,5)}$.

O objetivo deste estudo foi verificar a qualidade das prescrições de medicamentos sujeitos a controle especial dispensados em duas farmácias de natureza jurídica distintas de um município gaúcho. Também foi avaliada a legibilidade, o cumprimento dos procedimentos legais e os aspectos técnicos da prescrição para identificar quais os descumprimentos mais frequentes.

\section{MÉTODOS}

O estudo teve delineamento descritivo, transversal, quantitativo e retrospectivo, sendo aprovado pelo Comitê de Ética em Pesquisa da Universidade de Passo Fundo conforme Parecer 348.071 e protocolo CAAE No 18402713.4.0000.5342.

Os dados primários foram coletados das receitas e notificações de receitas retidas em duas farmácias de um município do interior do Estado do Rio Grande do Sul, sendo uma de natureza jurídica pública e outra privada. Estes estabelecimentos foram selecionados por representarem os pontos de atenção à saúde de maior procura da população.

O município avaliado fica localizado na região nordeste do Rio Grande do Sul e possui aproximadamente 5.000 habitantes, principalmente de origem italiana e polonesa, tendo na atividade agropecuária sua principal fonte de renda.

O período de coleta de dados foi de setembro a dezembro de 2012, totalizando quatro meses, por meio de uma amostra de conveniência, elegida por representar os serviços de maior demanda no município. 


\section{- Revista Eletrônica}

\section{RODRIGUES et al.}

REF-ISSN1808-0804 Vol.XII (1), 44-59, 2015.

As prescrições receberam codificação numérica e seus dados foram compilados em uma planilha, contendo três grupos de variáveis: legibilidade, cumprimento dos procedimentos legais e aspectos técnicos da prescrição. Estas variáveis são estabelecidas pela Organização Mundial da Saúde como parâmetros de boa prescrição ${ }^{(6)}$. Cada prescrição foi incluída no banco de dados individualmente, mesmo sendo da mesma pessoa, porém em datas diferentes e/ou dispensadas por farmácias distintas.

A legibilidade foi avaliada pela leitura da prescrição por dois pesquisadores independentes. Quando havia discordância entre as opiniões era realizada uma terceira avaliação na busca de consenso.

O cumprimento dos procedimentos legais foi atendido quando estavam presentes no receituário as seguintes características: nome completo do paciente, data da prescrição, assinatura do prescritor, número do registro profissional do prescritor $\mathrm{e}$ denominação do medicamento conforme a Denominação Comum Brasileira (DCB).

Os aspectos

técnicos presentes

receituário considerados para uma prescrição de qualidade foram a dose, a frequência, a via de administração e a forma farmacêutica(7).

Como desfecho primário do estudo buscou-se saber quais os descumprimentos das prescrições mais frequentes, no geral e pelos três grupos de variáveis, tendo como referencia os parâmetros da boa prescrição estabelecidos pela Organização Mundial da Saúde. A prescrição foi interpretada como completa quando era legível e preenchia todos os requisitos legais e técnicos de maneira satisfatória. Ao contrário disso, qualquer não conformidade classificava a prescrição como incompleta.

A análise dos resultados foi realizada por meio de estatística descritiva e inferencial. Para variáveis qualitativas foi aplicado o teste quiquadrado e, para as quantitativas, o teste T-Student. Para verificar a independência entre as variáveis de associação de descumprimento de prescrição e critérios relacionados, foi utilizado um teste não paramétrico com um nível de significância de 5\% por meio de um programa de estatístico (Statistical Package for the Social Sciences -SPSS - versão 18.0). 
RODRIGUES et al.

REF-ISSN1808-0804 Vol.XII (1), 44-59, 2015.

Todas as despesas relacionadas ao desenvolvimento da pesquisa foram de inteira responsabilidade dos pesquisadores e informamos que não há conflito de interesses.

\section{RESULTADOS E DISCUSSÃO}

No período de setembro a dezembro de 2012 foram dispensadas 889 prescrições relativas a Portaria 344/98 nas duas farmácias abrangidas pelo estudo, o número de prescrições analisadas foi diminuindo gradativamente conforme as perdas relacionadas a ilegibilidade e/ou incompletude dos dados conforme o fluxograma apresentado na Figura 1. A frequência dos medicamentos mais dispensados foi calculada a partir da analise de 683 receituários. E a qualidade das prescrições e a frequência dos descumprimentos foram averiguadas em 330 documentos. com média mensal de 222,2 prescrições, na proporção de 2:1 entre a farmácia privada e a farmácia pública da rede municipal de saúde (Figura 1).

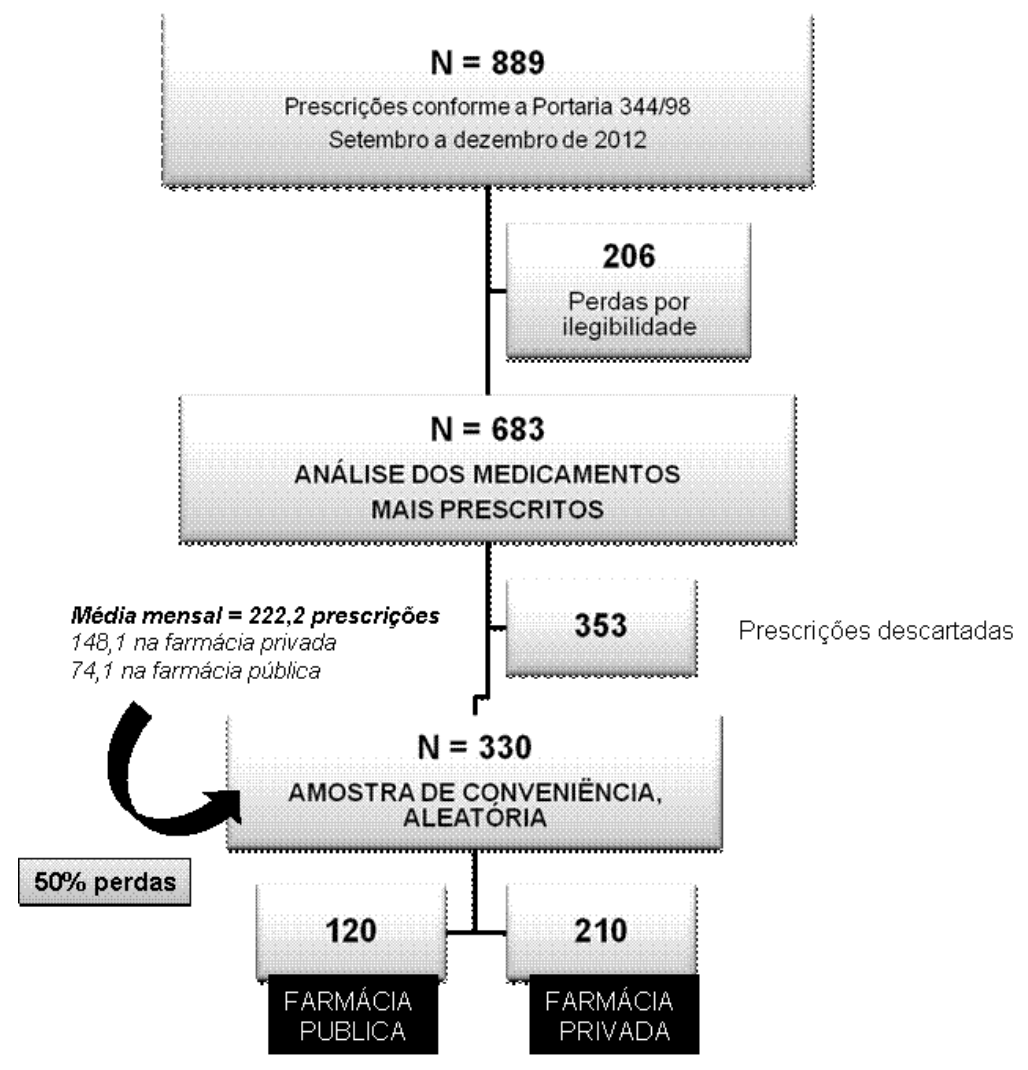


RODRIGUES et al.

REF-ISSN1808-0804 Vol.XII (1), 44-59, 2015.

Figura 1. Representação esquemática do fluxograma adotado para definição da amostra aleatória das prescrições inseridas no estudo, referente ao período de setembro a dezembro de 2012.

De acordo com a Portaria 344/1998, os medicamentos prescritos pertenciam às listas A3, B1 e C1 (Quadro 1).

Quadro 1. Distribuição dos 683 medicamentos dispensados conforme o tipo de receituário e a natureza jurídica da unidade dispensadora, com a identificação dos medicamentos mais prescritos em cada uma destas categorias.

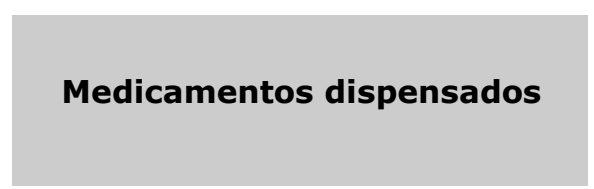

Total

Farmácia pública

Medicamento mais prescrito

Farmácia privada

Medicamento mais prescrito

Quanto aos medicamentos mais dispensados houve diferença entre os dois estabelecimentos, contudo a fluoxetina esteve entre os três medicamentos mais dispensados independente da natureza jurídica da

\section{Outras substâncias} sujeitas a controle especial

498 (72,9\%) 226 Fluoxetina $(n=39 ; 10,4 \%)$ 272 Fluoxetina $(n=45 ; 11,3 \%)$ farmácia. Já com relação aos medicamentos psicotrópicos, o clonazepam foi o mais dispensado na farmácia pública, enquanto que na farmácia privada foi a sertralina (Figuras 2 e 3 ).

\section{Psicotrópicos}

$185(27,1 \%)$

Clonazepam

$(n=60 ; 16,0 \%)$

90

Sertralina

$$
(n=41 ; 10,3 \%)
$$


RODRIGUES et al.

REF-ISSN1808-0804 Vol.XII (1), 44-59, 2015.

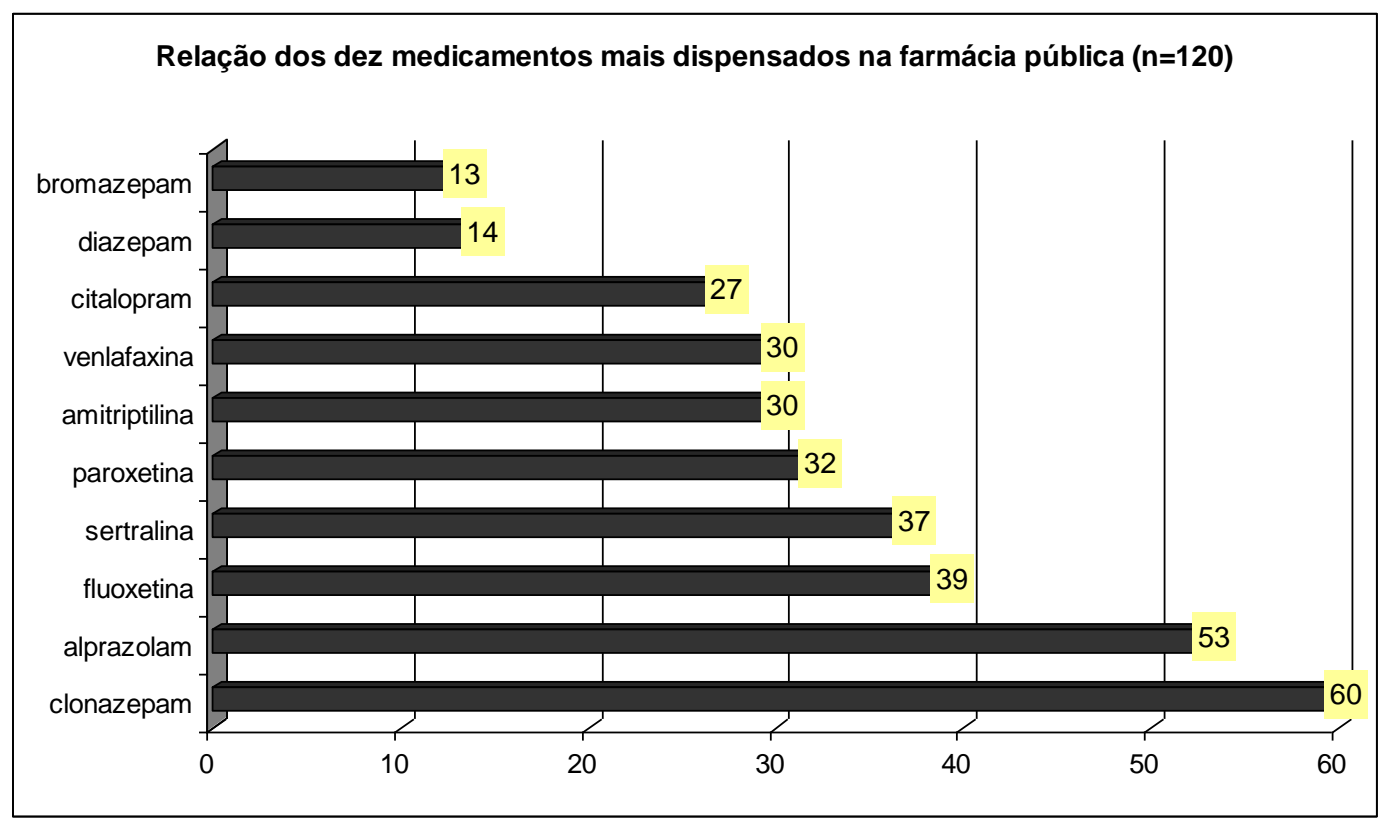

Figura 2. Frequência absoluta dos dez medicamentos mais dispensados na farmácia pública do município gaucho avaliado, no período de setembro a dezembro de 2012, a partir da analise aleatória de 120 prescrições.

A partir destes indicadores foram selecionadas aleatoriamente 120 prescrições da farmácia pública e 210 retidas na farmácia privada para verificar a qualidade da prática de prescrição, a análise dos indicadores de qualidade da boa prescrição e a verificação de descumprimentos de prescrição foram realizadas para amostra de 330 receituários. 
RODRIGUES et al.

REF-ISSN1808-0804 Vol.XII (1), 44-59, 2015.

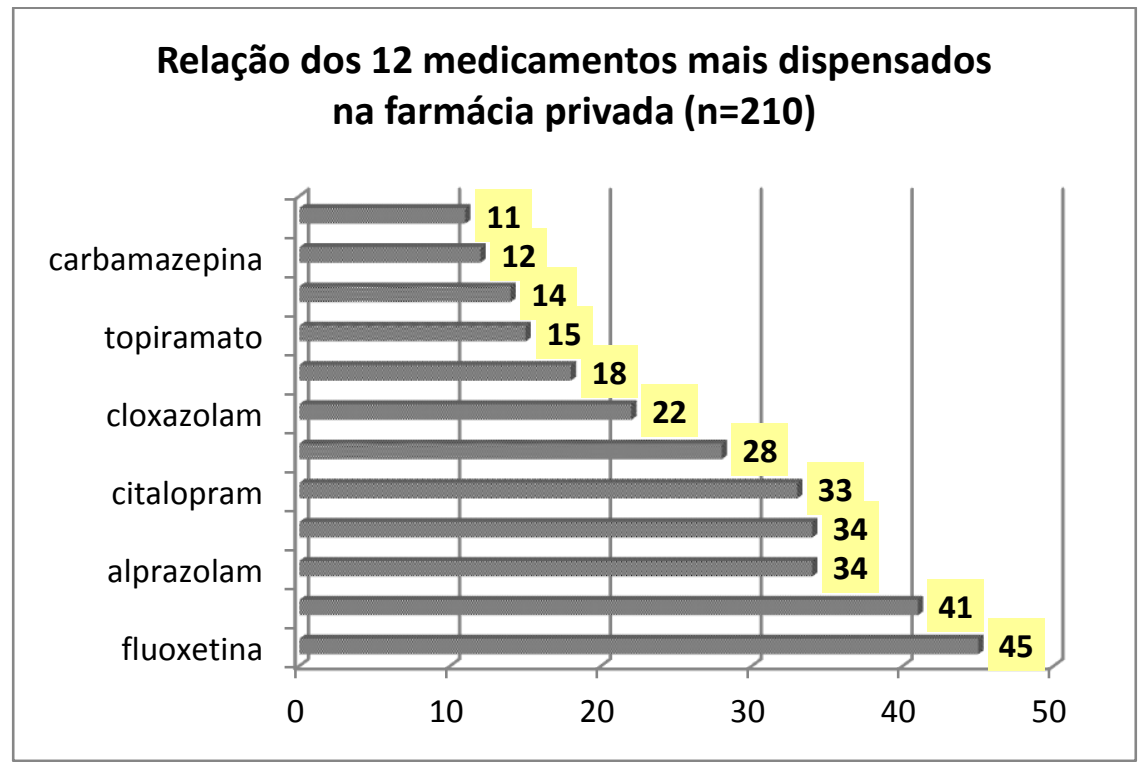

Figura 3. Frequência absoluta dos dez medicamentos mais dispensados na farmácia privada do município gaucho avaliado, no período de setembro a dezembro de 2012, a partir da analise aleatória de 210 prescrições.

A análise das 330 prescrições revelou predomínio de receituários para substancias entorpecentes (A1), seguido dos psicotrópicos (B2), conforme ilustra a Tabela 2. Salientamos que houve uma perda $(0,3 \%)$ pois o formulário usado para a dispensação não era o adequado para o tipo de medicamento prescrito.

Tabela 2. Distribuição dos medicamentos dispensados conforme o tipo de receituário e de unidade dispensadora para as 330 prescrições analisadas no estudo, tendo como referencia a Portaria 344/98.

\begin{tabular}{llll}
\hline Tipo de receituário & $\begin{array}{l}\text { Farmácia } \\
\text { pública* }\end{array}$ & $\begin{array}{l}\text { Farmácia } \\
\text { privada* }\end{array}$ & Total* \\
\hline Entorpecentes (A1) & 63 & 148 & 211 \\
\hline Psicotrópicos (B2) & 56 & 60 & 216 \\
\hline $\begin{array}{l}\text { Outras substancias sujeitas a } \\
\text { controle especial (C) }\end{array}$ & 1 & 1 & $\mathbf{3 2 9 * *}$ \\
\hline Total & $\mathbf{1 2 0}$ & $\mathbf{2 0 9}$ & \\
\hline
\end{tabular}

* Os números estão expressos em frequência absoluta.

**Houve uma perda pois o receituário estava inadequado ao tipo de medicamento prescrito conforme preconizado na Portaria 344/98.

O número de medicamentos prescritos por receituário variou de um a três (Tabela 3) e a maior parte foi dispensada para mulheres.

Destacamos que em 10,30\% das prescrições não foi possível identificar 


\section{RODRIGUES et al.}

REF-ISSN1808-0804 Vol.XII (1), 44-59, 2015.

o sexo do paciente a partir do nome

preenchido na prescrição.

Tabela 3. Frequência absoluta e relativa do número de medicamentos prescritos por receituário avaliados no período de setembro a dezembro de 2012 para o município avaliado.

\begin{tabular}{ccc}
\hline $\begin{array}{c}\text { Número de medicamentos } \\
\text { prescritos por receituário }\end{array}$ & $\begin{array}{c}\text { Freqüência absoluta } \\
(\mathbf{N})\end{array}$ & $\begin{array}{c}\text { Freqüência relativa } \\
(\mathbf{\%})\end{array}$ \\
\hline Um & 299 & 90,9 \\
Dois & 23 & 7,0 \\
Três & 7 & 2,1 \\
\hline Total & $\mathbf{3 2 9}$ & $\mathbf{1 0 0}$ \\
\hline
\end{tabular}

Ao analisar os aspectos isolados das prescrições, conforme a Portaria n. ${ }^{0} 344 / 1998$, verificamos que o nome do paciente esteve presente em

A data da prescrição foi outro item analisado e esteve presente em apenas $32,6 \%$ dos receituários (Tabela 4 ).

Tabela 4. Descrição da qualidade das 330 prescrições analisadas para o grupo de variáveis relacionados ao cumprimento dos procedimentos legais.

\begin{tabular}{lcc}
\hline Aspecto avaliado & $\begin{array}{c}\text { Frequência } \\
\text { absoluta } \\
(\mathrm{n})\end{array}$ & $\begin{array}{c}\text { Frequência } \\
\text { relativa } \\
(\%)\end{array}$ \\
\hline Nome completo do paciente & 293 & 88,8 \\
Presença da data da prescrição & 107 & 32,6 \\
$\begin{array}{l}\text { Presença da assinatura do prescritor } \\
\text { Presença do número do registro profissional do prescritor }\end{array}$ & 319 & 96,7 \\
$\begin{array}{l}\text { Medicamento denominado conforme a Denominação } \\
\text { Comum Brasileira (DCB) }\end{array}$ & 255 & $\mathbf{7 7 , 3}$ \\
\hline $\begin{array}{l}\text { Desempenho completamente adequado quanto ao } \\
\text { cumprimento dos procedimentos legais }\end{array}$ & $\mathbf{4 5 1}$ & $\mathbf{4 9 , 7}$ \\
\hline
\end{tabular}

Quanto a assinatura e o número do registro profissional do prescritor foram aspectos presentes em mais de $70 \%$ das prescrições, sendo a assinatura a informação mais frequente nos 330 receituários analisados, apesar de não constar em 11 prescrições (Tabela 4).

A denominação do medicamento no receituário seguiu a Denominação
298 prescrições $(90,3 \%)$. As demais prescrições apresentaram nome incompleto, ilegível ou sem nome. 


\section{RODRIGUES et al.}

REF-ISSN1808-0804 Vol.XII (1), 44-59, 2015.

técnicos) no geral houve $39,4 \%$ de prescrições adequadas.

Foram realizados testes de associação para verificar se o tipo de unidade dispensadora interferia na qualidade das prescrições (Tabela 5). $\mathrm{Na}$ legibilidade dos receituários não houve diferença estatística significativa de legibilidade, em relação a natureza jurídica da farmácia dispensadora $(p=0,067)$, bem como os aspectos técnicos da prescrição $(p=0,559)$ e a qualidade no geral dos receituários $(p=0,443)$, avaliada pelos três grupos de variáveis. Contudo, ao comparar o cumprimento dos procedimentos legais verificamos diferença significativa entre as farmácias e os receituários foram mais adequados na farmácia privada $(p=0,048)$.

Tabela 5. Comparação entre a frequência da qualidade das 330 prescrições analisadas nas duas unidades dispensadoras, tanto nos três grupos de características avaliadas como no aspecto geral, denominado de prescrição completa.

\begin{tabular}{|l|c|c|c|c|c|c|}
\hline \multirow{2}{*}{ Variáveis } & \multicolumn{2}{|c|}{$\begin{array}{c}\text { Farmácia pública } \\
(\mathbf{n = 1 2 0 )}\end{array}$} & $\begin{array}{c}\text { Farmácia privada } \\
(\mathbf{n = 2 1 0 )}\end{array}$ & \multicolumn{2}{c|}{ Total } \\
\cline { 2 - 7 } & $\mathrm{N}^{*}$ & $\%{ }^{* *}$ & $\mathrm{~N} *$ & $\%{ }^{* *}$ & $\mathrm{~N}^{*}$ & $\%^{* *}$ \\
\hline Legibilidade & 92 & 76,7 & 182 & 86,7 & 274 & 83,0 \\
\hline $\begin{array}{l}\text { Cumprimento dos } \\
\text { procedimentos legais }\end{array}$ & 51 & 42,5 & 113 & 53,8 & 164 & 49,7 \\
\hline $\begin{array}{l}\text { Aspectos técnicos da } \\
\text { prescrição }\end{array}$ & 85 & 70,8 & 155 & 73,8 & 240 & 72,7 \\
\hline Prescrição completa & 44 & 36,7 & 86 & 41,0 & 130 & 39,4 \\
\hline
\end{tabular}

*N: representa a frequência absoluta.

$* * \%$ : representa a frequência relativa.

É importante conhecer o perfil de prescrição e dispensação de psicotrópicos para situar os prescritores, pacientes e serviços farmacêuticos para o uso racional destes medicamentos, pois de acordo com a Organização Mundial da Saúde mais de $50 \%$ de todos os medicamentos são incorretamente prescritos, dispensados e vendidos; e mais de $50 \%$ dos pacientes fazem o uso de forma incorreta ${ }^{(8)}$.

Como benefício de uma boa prescrição pode-se minimizar as reações adversas, otimizar a adesão ao tratamento e, além disso, qualificar o cuidado em saúde. Somado a isso, e a presença do farmacêutico, a boa prescrição garante a dispensação correta do medicamento e ao prescritor, maior credibilidade frente ao paciente e ao farmacêutico. 


\section{- Revista Eletrônica}

\section{RODRIGUES et al.}

REF-ISSN1808-0804 Vol.XII (1), 44-59, 2015.

Além disso, um tema bastante discutido tem sido a segurança do paciente pois grande parte dos eventos adversos ocorridos em decorrência da assistência à saúde, especialmente no ambiente hospitalar são por causas evitáveis ${ }^{(9-11)}$.

Entre os medicamentos mais usados mundialmente estão os benzodiazepínicos, havendo estimativas que entre 1 e $3 \%$ de toda a população ocidental já os tenha consumido por mais de um ano(12-13). O benzodiazepínico mais prescrito no município avaliado foi o clonazepam, o qual teve maior dispensação pela farmácia pública.

O antidepressivo mais prescrito neste estudo foi a fluoxetina, semelhante a outros trabalhos. Sabese que este antidepressivo é atualmente um dos mais prescritos no Brasil e no mundo, havendo indícios de que possa atuar na de perda de peso durante vários meses após o início da terapia ${ }^{(5,14-15)}$.

Carlini et $\mathrm{al}^{16}$ ao analisar mais de 30.000 receitas especiais em Santo André, SP, verificou que as prescrições de fluoxetina eram predominantemente destinadas a mulheres $(79,8 \%)$. Na maioria dos casos, a fluoxetina foi prescrita em associação com outras substâncias ativas, inclusive anfetaminas anoréticas, sinalizando que a utilização de fluoxetina teria um fim estético (perda de peso), e não um fim terapêutico (tratamento de depressão).

No município avaliado neste estudo pode ser que esta característica seja um dos fatores do consumo elevado de fluoxetina.

Esta pesquisa também revelou que apenas $39,4 \%$ das prescrições estavam adequadas e completas quando considerado a sua legibilidade, procedimentos legais e aspectos técnicos. Resultados semelhantes foram verificados em um estudo realizado em município do meio oeste de Santa Catarina, no qual $82 \%$ das prescrições continham algum tipo de descumprimento(17). Isso deixa claro que erros nas prescrições não estão apenas presentes no município gaúcho investigado, e leva a crer que tais descumprimentos são comuns em outros lugares do país.

Em Ribeirão Preto, SP, também foram verificados percentuais significativos de ilegibilidade na análise de 753 receituários de psicotrópicos das listas B e C1 e as falhas mais frequentes foram na identificação do paciente ${ }^{(3)}$. 
RODRIGUES et al.

REF-ISSN1808-0804 Vol.XII (1), 44-59, 2015.

Ao analisar a prática da prescrição de medicamentos de alto risco e sua relação com a prevalência de erros de medicação em um hospital de Minas Gerais, os pesquisadores identificaram que erros no nome do paciente, dificuldades na identificação do prescritor e pouca legibilidade ou prescrições ilegíveis foram as não conformidades mais frequentes $^{(11)}$.

Esta realidade pode sugerir certo descaso por parte do prescritor já que entrega ao paciente uma prescrição incompleta. Isso pode levar à incorreta adesão ao tratamento por parte do paciente e equívocos na dispensação por parte dos farmacêuticos.

Outra pergunta que se deve fazer é se o médico realmente conhece a legislação que regulamenta o correto preenchimento da prescrição. Em contrapartida, o farmacêutico também leva uma parcela de culpa, pois não deve aceitar uma receita incompleta na hora de dispensar um medicamento de controle especial.

\section{CONCLUSÃO}

O uso irracional e o consumo elevado de psicotrópicos pode comprometer a segurança do paciente. Por meio desta pesquisa foi possível conhecer qual o medicamento e classe farmacológica mais utilizada pela população do município investigado, bem como os descumprimentos mais encontrados nas prescrições. Como resultado deste estudo, busca-se garantir maior segurança e informação para os pacientes que utilizam medicamentos psicotrópicos.

A qualidade das prescrições de medicamentos sujeitos a controle especial dispensados no município avaliado está comprometida, especialmente pelo descumprimento de exigências legais, onde foram detectados os descumprimentos mais frequentes. A legibilidade das prescrições teve um desempenho superior a de outros estudos, embora parte dos receituários não puderam ser decifrados, podendo comprometer o entendimento do paciente ou do profissional responsável pela dispensação do medicamento.

\section{AGRADECIMENTOS}

Agradecemos aos serviços de farmácia que cederam as prescrições para realização do estudo. 
RODRIGUES et al.

REF-ISSN1808-0804 Vol.XII (1), 44-59, 2015.

\section{REFERÊNCIAS}

1. Brasil, Ministerio da Saúde. Portaria 344, de 12 de maio de 1998. R. T. Sobre substâncias e medicamentos sujeitos a controle especial. Convenção Única Sobre Entorpecentes,. v. 1961, N. C, 1998.

2. Néri EDR. Erros de prescrição de medicamentos em um hospital brasileiro. Revista Associação Médica Brasileira, 2011.

3. Andrade MF, Célia RA, Santos VG. Prescrição de psicotrópicos : avaliação das informações contidas em receitas e notificações. Revista Brasileira Ciencia Farmacêutica., 2004, v. 40.

4. Sadock BJ, Sadock VA. Manual de farmacologia psiquiátrica de Kaplan e Sadock. 3.ed. Porto Alegre: Artmed, 2002. 376 p.

5. Rang HP, Dale MM, Ritter JM. Farmacologia.4. ed. Rio de Janeiro: Guanabara Koogan, 2001. 703 p.

6. Organização Mundial da Saúde. Guia Para A Boa Prescrição Médica. Porto Alegre: Artmed, 1998. 124p.

7. Brasil, Ministerio da Saúde. Avaliação da Assistência Farmacêutica no Brasil: Estrutura, Processo e Resultados. Brasília - DF, 2005.

8. Rérat $C$, Nascimento Júnior, JM, Wannmacher L (Coord.). Uso racional de medicamentos: temas relacionados. Brasília, DF: Ministério da Saúde, 2012. 154 p.

9. Brennan TA, Leape LL, Laird NM, Hebert L, Localio AR, Lawthers AG, et al. Incidence of adverse events and negligence in hospitalized patients: results of the Harvard Medical Practice Study I. N Engl J Med. 1991;324(6):370-6.

10. Leape LL, Brennan TA, Laird N, Lawthers AG, Localio AR, Barnes BA, et al. The nature of adverse events and negligence in hospitalized patients. Results of the Harvard Medical Practice Study II. N Engl J Med. $1991 ; 324(6): 377-84$.

11. Rosa MB. Erros na prescrição hospitalar de medicamentos potencialmente perigosos. Rev. Saúde Pública [online]. 2009, vol.43, n.3, pp. 490-498.

12. Baldessarini RJ Drugs And The Treatment Of Psychiatric Disorders: Psychosis And Anxiety. In: Hardman JG, Gilman AG, Limbird LE. Eds. Goodman \& Gilman'S The Pharmacological Basis Of Therapeutics. 9 Ed. New York: Mcgraw Hill, 1995. Cap. 18, P. 399 - 430.

13. Huf G, Lopes CS, Rosenfeld S. O uso prolongado de benzodiazepínicos em mulheres de um centro de convivência para idosos. Cad. Saúde Pública, Rio De Janeiro, V. 16, N. 2, P. 351-62, 2000.

14. Mota DM, Silva-Jr GG. Evidências advindas do consumo de medicamentos moduladores do apetite no Brasil: um estudo farmacoeconométrico. Rev. Assoc. Med. Bras. [serial on the Internet]. 2012 Feb [cited 2013 Dec 04] ; 58( 1 ): 88-94. 
RODRIGUES et al.

REF-ISSN1808-0804 Vol.XII (1), 44-59, 2015.

15. Istilli PT et al. Antidepressivos: uso e conhecimento entre estudantes de enfermagem. Rev. Latino-Am. Enfermagem [online]. 2010, vol.18, n.3, pp. 421-428.

16. Carlini EA et al. Fluoxetina: indícios de uso inadequado. J. bras. psiquiatr. [online]. 2009, vol.58, n.2, pp. 97-100.

17. Lins BG, Cazzamalli F, Zancanaro V. Análise de erros nas prescrições médicas de uma unidade básica de um município do meio oeste catarinense. Revista Interdisciplinar de Estudos em Saúde. V.1, n.2, p 62-77, 2012. 